\title{
Adenovirus-mediated shRNAs for co-repression of miR-221 and miR-222 expression and function in glioblastoma cells
}

\author{
XUAN WANG ${ }^{1-5^{*}}$, LEI HAN $^{1-3^{*}}$, ANLING ZHANG ${ }^{2,3}$, GUANGXIU WANG ${ }^{2,3}$, ZHIFAN JIA ${ }^{2,3}$, \\ YANG YANG ${ }^{1-3}$, XIAO YUE ${ }^{1-3}$, PEIYU PU ${ }^{1-3}$, CHANGHONG SHEN $^{1}$ and CHUNSHENG KANG ${ }^{1-3}$ \\ ${ }^{1}$ Department of Neurosurgery, Tianjin Medical University General Hospital; ${ }^{2}$ Laboratory of Neuro-Oncology, \\ Tianjin Neurological Institute; ${ }^{3}$ Key Laboratory of Neurotrauma, Variation and Regeneration, Ministry of Education and \\ Tianjin Municipal Government, Tianjin; ${ }^{4}$ Department of Neurosurgery, Fuxing Hospital, Capital Medical University, \\ Beijing; ${ }^{5}$ Department of Neurosurgery, Sanbo Brain Institute, Capital Medical University, Beijing, P.R. China
}

Received May 19, 2010; Accepted August 13, 2010

DOI: $10.3892 /$ or_00001047

\begin{abstract}
Aberrantly expressed miRNAs are linked to the regulation of oncogenes and/or tumor suppression genes within the cell signal transduction pathway network, thereby contributing to carcinogenesis. miRNA function can be antagonized, thus representing a novel anti-tumor approach for integrated cancer therapy. In this study, we designed adenovirally-expressed shRNAs that functionally co-repressed the expression of miR-221 and miR-222, which are related to glioblastoma, to overcome the low efficiency of gene therapy. In addition, we generated novel shRNAs whose 3 ' ends were mutated in the region complementary to the target miRNA's 5 ' seed region to reduce the stability of binding with the miRNA. Various inhibition levels of miRNA were achieved: classic shRNAs yielded the greatest reduction in miRNA levels, followed by mutated shRNAs and the blank control, as determined by qRT-PCR. These results were confirmed by the protein expression of $\mathrm{p} 27^{\mathrm{kip} 1}$, the validated target of miR$221 / 222$, the effect on cell cycle arrest in $G_{1}$ phase, and the impact on cell apoptosis. These results suggested that we could produce shRNAs encoded by adenovirus that co-repressed multiple tumor-related miRNAs simultaneously, and that the level of repression and the effect on the function of a specific miRNA could be achieved in a semi-quantitative manner.
\end{abstract}

Correspondence to: Professor Changhong Shen, Department of Neurosurgery, Tianjin Medical University General Hospital, Tianjin 300052, P.R. China

E-mail: osen1984@gmail.com

Professor Chunsheng Kang, Laboratory of Neuro-Oncology, Tianjin Neurological Institute, Tianjin 300052, P.R. China

E-mail: kang97061@yahoo.com

*Contributed equally

Key words: glioma, miR-221, miR-222, shRNA, adenovirus vector, gene therapy

\section{Introduction}

MicroRNAs (miRNAs) are a set of small single stranded noncoding RNA molecules that serve as important negative gene regulators (1). Partial complementary pairing between the 3'-untranslated region (3'UTR) of the messenger RNA (mRNA) and the 5 ' seed region of a miRNA is essential for the post-transcriptional modulation of the target gene expression (2). Given the diversity and abundance of target genes, miRNAs appear to functionally interact with various components of cellular signal transduction pathways known as tumor suppressor genes or oncogenes (3) to generate a complex combinatorial network. Examination of the network profiles has revealed widespread deregulation of these molecules in diverse cancers (4), suggesting that miRNAs play critical roles in carcinogenesis (4).

Short hairpin RNAs (shRNAs) are novel modulators that act via RNA interference (RNAi) (5), which contains the pre-designed antisense and sense sequence separated by a loop structure. shRNAs are believed to be processed into their functional form in cells, where the antisense strand functions as a 'guide' molecule that leads to an activated form of RISC after the sense strand departure as a 'passenger' molecule (Fig. 1). shRNAs can function to antagonize aberrantly expressed miRNAs resulting in tumor suppression (6). Thus, this technique is a potential improvement compared with tumor gene therapy by conventional RNAi or antisense oligonucleotide. However, in a recent study, shortcomings were reported in the application of lentiviral vectors, and shRNAs are designed to target single miRNAs, not the entire set of aberrant miRNAs and miRNA clusters pertaining to a certain category of cancer.

Ciafrè $e t a l$ and our previous work used a microarray method to show that the expression of the miR-221/222 cluster was strongly upregulated in glioblastoma $(7,8)$. Recently, we demonstrated that co-suppression of miR-221 and miR-222 expression by oligonucleotides might inhibit glioblastoma cell proliferation by directly upregulating the tumor suppressor gene $\mathrm{p} 27^{\mathrm{kip} 1}$ in vivo and in vitro (9). In the current study, we produced an expression cassette embedding two shRNAs to co-repress the expression of miR-221/222 in glioblastoma cells, introduced using a recombinant 


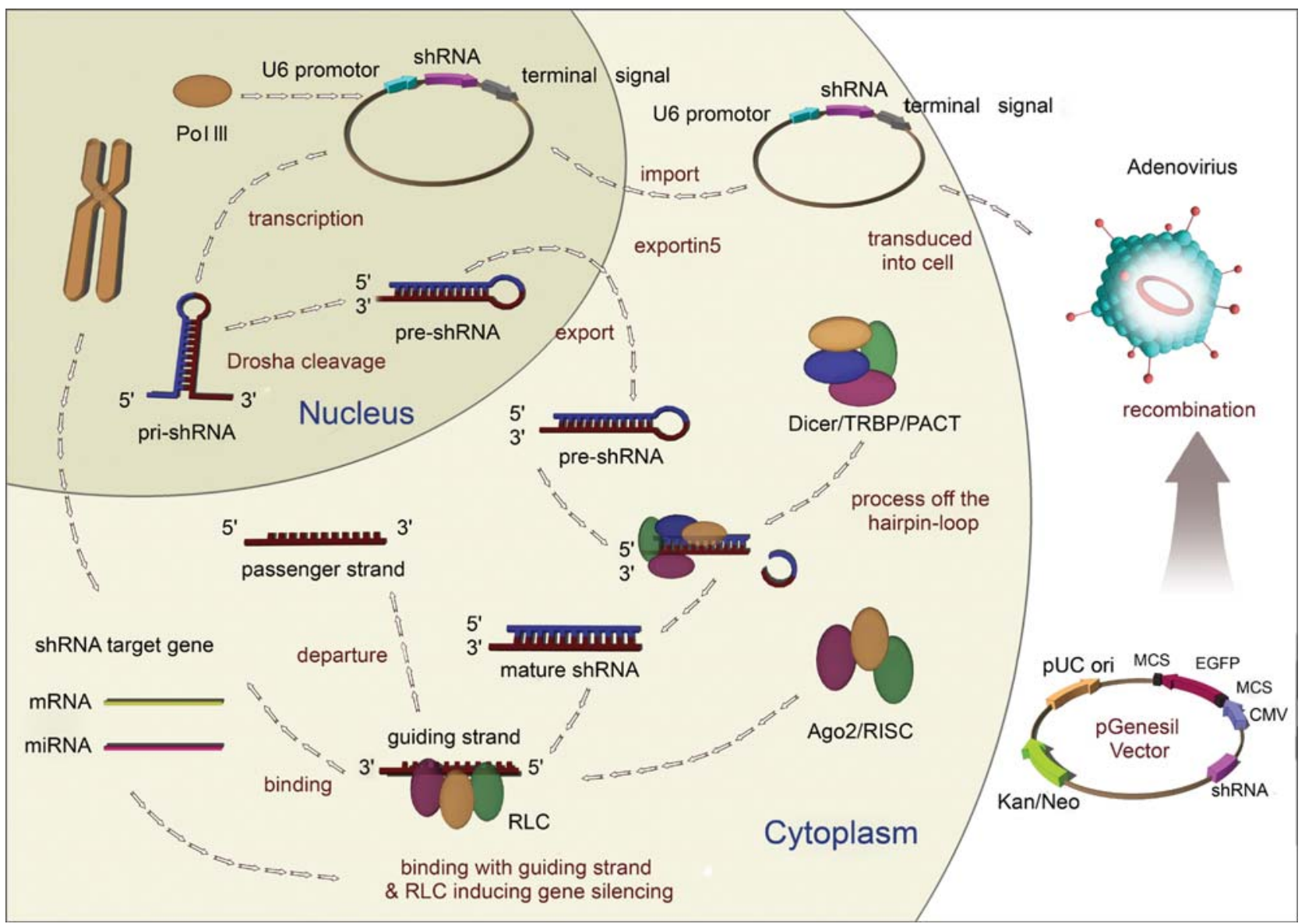

Figure 1. Schematic of the shRNA-mediated RNA interference pathway. After exogenous (artificial) introduction into the cytoplasm, the shRNA expression vector needs to be transported into the nucleus for transcription by RNA polymerase III via the U6 promoter. The primary transcript (pri-shRNA) follows a similar route as that of the primary transcript of a miRNA. After processing in the nucleus to form pre-shRNA, the shRNA is loaded onto the TRBP/PACT/Dicer complex in the cytoplasm where it is further processed to mature shRNA. After recruiting the Ago2 protein containing RISC and passenger strand (sense sequence) departure, the guiding strand (sense sequence) leads the RLC (RISC-loading complex) to specifically bind to the target RNA molecule and function via RNAi.

adenoviral vector. Furthermore, we demonstrated the expression of the miRNA at distinct levels using a mutation strategy. Thus, we unraveled possible concomitant mechanisms of the structural interactions between shRNAs and target miRNAs.

\section{Materials and methods}

Synthesis of shRNAs and cloning of U6-shRNA expression cassettes. Four 23-nt antisense sequences were designed to produce shRNA expression cassettes. Antisense sequences of classic and mutated shRNAs corresponding to positions 65-87 of miR-221 and 69-92 of miR-222 are shown in Table I, as well as their complementary sense sequences, were chemically synthesized, including SacI restriction site overhang sequences for identification. In addition, each sense sequence harbored a $5^{\prime}$ tetraloop and a $3^{\prime}$ stretch of Ts as an RNA polymerase III transcription termination signal.

Linear vectors pGenesil-1.1 and pGenesil-1.2 were used as eukaryotic vectors, providing U6-promoters to the expression cassettes. pGenesil-1.1 was used for shmiR-221 and pGenesil-1.2 was used for shmiR-222 and the annealed shRNA sequences were ligated into them. The vector backbones and the structures of the shRNA expression
Table I. Target sequences of miR-221 and miR-222.

\begin{tabular}{lc}
\hline Name & Sequence (5'-3') \\
\hline miR-221 & 65 AGCUACAUUGUCUGCUGGGUUUC $\sim 87$ \\
miR-222 & 69 AGCUACAUCUGGCUACUGGGUCUC $\sim 92$ \\
\hline
\end{tabular}

cassettes are shown in Fig. 2. We generated four vectors containing shRNAs targeting miR-221 or miR-222, designated p1-mut-shmiR-221, p1-class-shmiR-221, p2-mut-shmiR-222, $\mathrm{p} 2$-class-shmiR-222. The detailed strategies are available from the authors on request.

Construction of U6-shRNA co-expression cassettes and preparation of recombinant adenoviral vectors. To produce the co-expression cassettes, the expression constructs p1-mutshmiR-221, p1-class-shmiR-221, p2-mut-shmiR-222, and p2class-shmiR-222 were initially digested with HindIII/BamHI. The large fragments of p1-mut-shmiR-221 or p1-class-shmiR221 (encoded by pGenesil1.1) and the small fragments of p2-mut-shmiR-222 or p2-class-shmiR-222 (encoded by pGenesil1.2) were then ligated together, yielding p-mut-shmiR- 


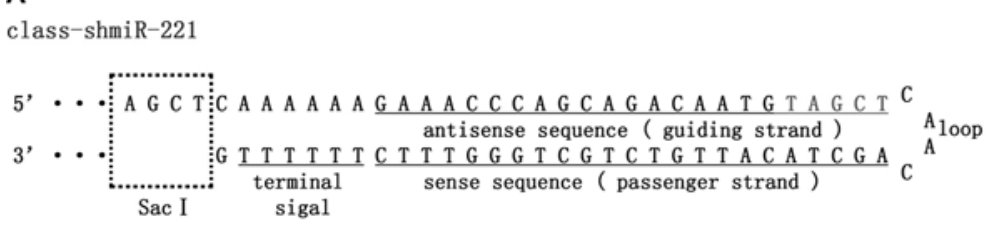

B

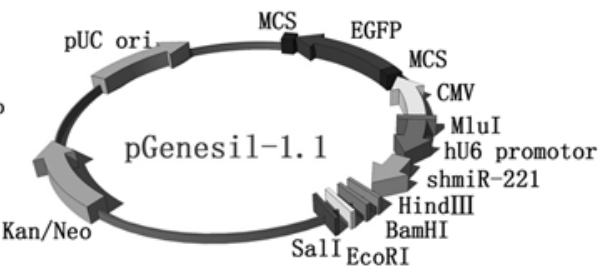

mut-shmiR-221

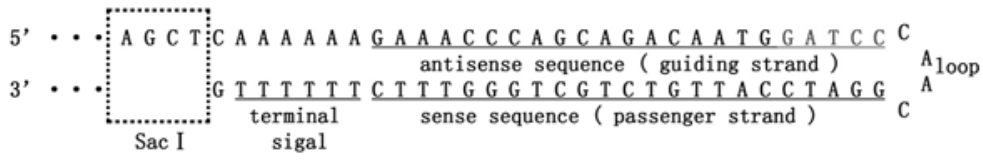

C

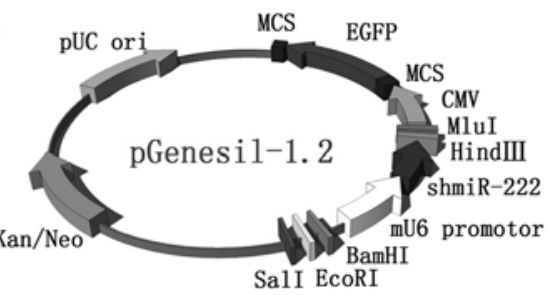

class-shmiR-222

5, ... AGCT CAAAAAAGAGACCCAGTAGCCAGATGTAGCT T

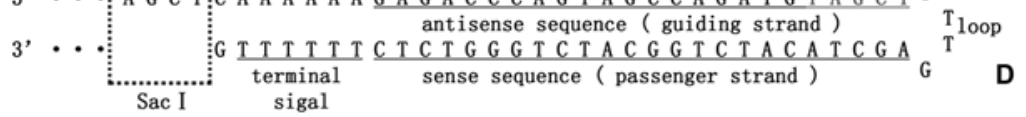

SalI EcoRI

pUC ori MCS EGFP

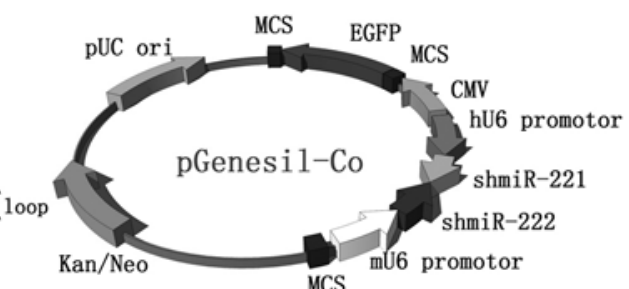

Figure 2. Schematic representation of eukaryotic plasmid vectors harboring shRNAs. (A) Four shmiRNA expression cassettes that form hairpins, including mutated and classic types. The $3^{\prime}$ end bases of the antisense sequence (guiding strand) complementary to the target miRNA 5' seed region are shown in light font. (B) pGenesil-1.1 vector encoding shmiR-221. (C) pGenesil-1.2 vector encoding shmiR-222. (D) pGenesil-Co vector composed of pGenesil-1.1 and pGenesil-1.2 that encode shmiR-221 and shmiR-222.

221/222 and p-class-shmiR-221/222, respectively. The final backbone of the co-expression vector, designated pGenesilCo, is shown in Fig. 2D. The correct sequences were screened using single restriction endonuclease analysis and confirmed by DNA sequencing for each plasmid.

Adenoviral vectors encoding either mut-shmiR-221/222 or class-shmiR-221/222 were generated using the adenovirus expression system (Genesil, Wuhan, China) by homogeneous recombination resulting in Ad-mut-shmiR-221/222 or Adclass-shmiR-221/222, according to the manufacturer's protocol.

Preparation of recombinant adenoviral supernatants. Adenoviral particles were generated by HEK293 cells transfected with linearized recombinant adenoviral vectors, and confirmed by detection of the fluorescent expression cassette in the viral backbone. The viral supernatants were concentrated after amplification in HEK293 cells showing a cytopathic effect (CPE). The viral titer was determined by a plaque assay with serial dilutions of supernatants, and the titer was generally around $1-3 \times 10^{8} \mathrm{pfu} / \mathrm{cell}$.

Cell culture and adenoviral gene transduction. The human LN229 and U251 glioblastoma cell lines were obtained from the Institute of Biochemistry and Cell Biology, Chinese Academy of Sciences (Shanghai, China) and were maintained in Dulbecco's modified Eagle's medium (DMEM, Invitrogen,
Carlsbad, CA, USA) supplemented with $10 \%$ fetal bovine serum (Invitrogen), 2 mM L-glutamine (Sigma, St. Louis, MO, USA), 100 units of penicillin/ml (Sigma), and $100 \mu \mathrm{g}$ of streptomycin $/ \mathrm{ml}$ (Sigma), at $37^{\circ} \mathrm{C}$ with $5 \% \mathrm{CO}_{2}$.

The adenoviral supernatants were transduced into LN229 and $\mathrm{U} 251$ cells at $70 \%$ confluence per well in 6-well plates. Adenoviral transduction was performed with an MOI of $\sim 50$ for the blank control, mut-shmiRNA, and class-shmiRNA constructs.

RNA isolation and quantitative RT-PCR. Total RNA was isolated from GBM cells using the TRIzol reagent (Invitrogen). Expressions of mature miR-221/222 were determined by quantitative RT-PCR using the Hairpin-it ${ }^{\text {TM }}$ miRNAs qPCR quantitation kit (GenePharma, Shanghai, China). Reverse transcriptase reactions were incubated in a PTC-200 Thermal Cycler (Bio-Rad, Hercules, CA, USA) in a 96-well plate for $30 \mathrm{~min}$ at $16^{\circ} \mathrm{C}$, followed by $30 \mathrm{~min}$ at $42^{\circ} \mathrm{C}, 10 \mathrm{~min}$ at $85^{\circ} \mathrm{C}$, and then held at $4^{\circ} \mathrm{C}$. Real-time PCR was performed using a DNA Engine Opticon2 system (Bio-Rad). The following real-time PCR protocol was used: denaturation program $\left(95^{\circ} \mathrm{C}\right.$ for $\left.3 \mathrm{~min}\right)$, amplification and quantification program (40 cycles of $95^{\circ} \mathrm{C}$ for $12 \mathrm{sec}$ and $62^{\circ} \mathrm{C}$ for $40 \mathrm{sec}$ ), and melting curve program (from $62^{\circ} \mathrm{C}$ to $95^{\circ} \mathrm{C}$, read every $0.2^{\circ} \mathrm{C}$, hold $2 \mathrm{sec}$ ). U6 RNA (GenePharma) was used for normalization. Data are shown as fold change $(2-\Delta \Delta C \mathrm{CT})$, where $\Delta \Delta \mathrm{C}_{\mathrm{T}}=\left(\mathrm{C}_{\mathrm{TmiRNA}}-\mathrm{C}_{\mathrm{TU}}\right)_{\text {shmiRNA }}-\left(\mathrm{C}_{\mathrm{TmiRNA}}-\mathrm{C}_{\mathrm{TU}}\right)_{\mathrm{ctrl}}$. 
Western blot analysis. Equal amounts of protein per lane were separated by $8 \%$ SDS-polyacrylamide gel and transferred to PVDF membranes (Millipore, Bedford, MA, USA), then incubated with a primary antibody against $\mathrm{p} 27^{\mathrm{kip} 1}$ (CST, Danvers, MA, USA) followed by incubation with an HRPconjugated secondary antibody (Zymed, San Francisco, CA, USA). The specific protein was detected using a SuperSignal protein detection kit (Pierce, Rockford, IL, USA). The membrane was stripped and reprobed with an antibody against GAPDH (Santa Cruz Biotechnology, Santa Cruz, CA, USA) as a loading control. Proteins were detected with ChemiGenius2 via GeneSnap software (Syngene, Frederick, MD, USA). Densitometry was performed on scanned images using Quantity One software 4.6.2 (Bio-Rad).

Cell cycle analysis. Transduced and parental cells in the log phase of growth were harvested, washed with PBS, fixed with $75 \%$ ethanol overnight at $4^{\circ} \mathrm{C}$, and then incubated with RNase at $37^{\circ} \mathrm{C}$ for $30 \mathrm{~min}$. Nuclei of cells were stained with propidium iodide (PI) for $30 \mathrm{~min}$. A total of $10^{4}$ nuclei were examined in a FACS Calibur flow cytometer (BectonDickinson, Franklin Lakes, NJ, USA) and DNA histograms were analyzed by Modifit software. Results were presented as percentage of cells in each phase.

Caspase-Glo 3/7 assay. To measure apoptosis induced by the shRNAs, the activation of caspase-3/7 was analyzed using the Caspase-Glo ${ }^{\mathrm{TM}}$ 3/7 assay kit (Promega, Mannheim, Germany). Luciferase activity was recorded as relative light units per second (RLU) using a TriStar LB941 (Berthold, Bad Wildbad, Germany). Data reported represent the average of four wells. The 'no cell' control value was subtracted from the value for each well.

RNA complex conformation prediction. We used two computer programs, RNAfold and RNAeval, to predict (from the provided sequences) the tertiary structures and to calculate free energy states of guiding strand:miRNA complexes, respectively as described by Parisien et al (10). Using these two programs, we were able to compare the differences between these two kinds of complexes. The two algorithms are available from the authors on request.

Statistical analysis. Statistics was determined using ANOVA, a $\chi^{2}$ test, or $t$ test using SPSS13.0. Statistical significance was set at $\mathrm{P}<0.05$.

\section{Results}

Generation of co-modulating shRNA-encoding adenoviruses. The first objective of our research was to design adenoviral vector systems that could co-repress the related multiple miRNAs by encoding shRNAs. We produced two constructs in the pGenesil-Co vector comprising pGenesil-1.1 and pGenesil-1.2 that encoded shmiR-221 and shmiR-222, respectively (Fig. 2). The corresponding adenoviral vector was produced after recombination.

The second objective was to achieve different modulation effects of specific miRNAs. To accomplish this, we designed a series of specific shRNAs, which could reduce miRNA
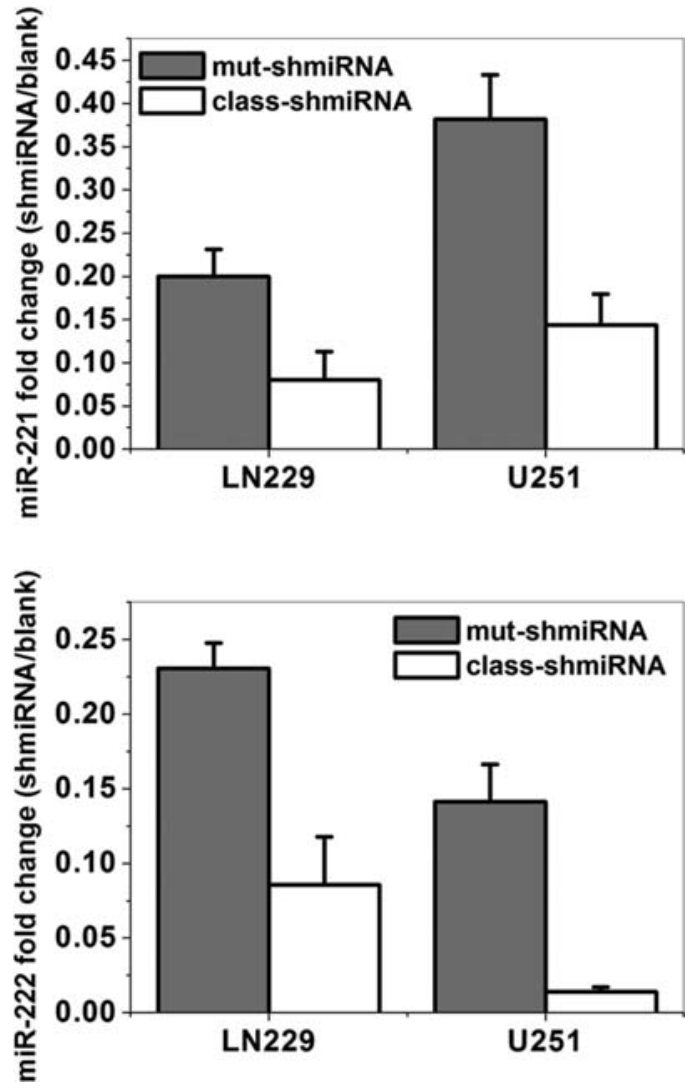

Figure 3. qRT-PCR of miRNAs in the presence of Ad-mut-shmiR-221/222 and Ad-class-shmiR-221/222, respectively. The histograms indicate foldchanges of miR-221/222 levels as determined by miR-qRT-PCR, normalized in comparison with blank controls.

expression distinctly, including perfect and partial complementary designs referred to as mut-shmiR-221, classshmiR-221, mut-shmiR-222 and class-shmiR-222 (Fig. 2). The principle of the design of the partially complementary shRNAs was to mutate the 3 '-end bases that were complementary to the target miRNA's 5'-seed region.

Finally, we obtained two adenoviral vectors, one encoded mut-shmiR-221 and mut-shmiR-222, the other encoded classshmiR-221 and class-shmiR-222, referred as Ad-mut-shmiR221/222 and Ad-class-shmiR-221/222 or mut-shmiRNA and class-shmiRNA for simplicity.

miRNA expression in the presence of adenovirally encoded shRNAs. On the basis of generation of mut-shmiRNA and class-shmiRNA, we next validated whether adenoviral vectorencoded shRNAs could silence miR-221 and miR-222 expression to different degrees in LN229 and U251 cells. The knockdown efficiencies of the mutated and classic shRNAs were measured by quantification of miR-221 and miR-222 expression using qRT-PCR. As shown in Fig. 3, all AdshRNAs caused significant repression of miR-221 and miR222, but Ad-mut- shmiR-221/222 (miR-221: 31\% in LN229, $41 \%$ in U251; miR-222: $26 \%$ in LN229, $12 \%$ in U251) was less efficient compared with Ad-class-shmiR-221/222 (miR221: $12 \%$ in LN229, $14 \%$ in U251; miR-222: $9 \%$ in LN229, $2 \%$ in U251) $48 \mathrm{~h}$ after transduction, as compared to blank control cells. 
A

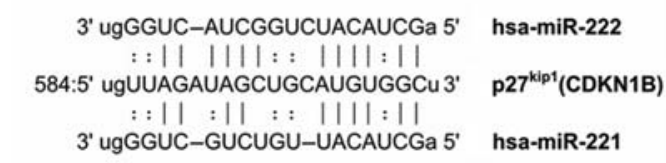

B

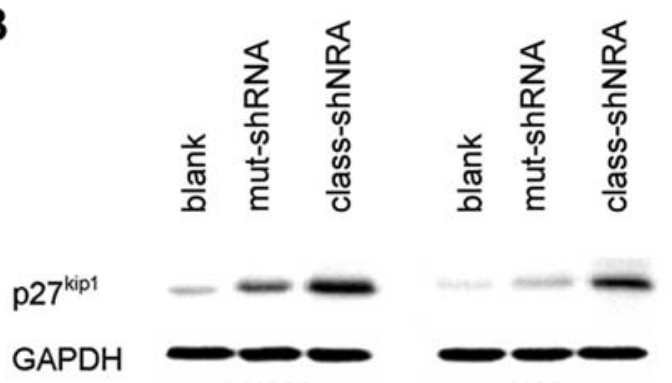

C
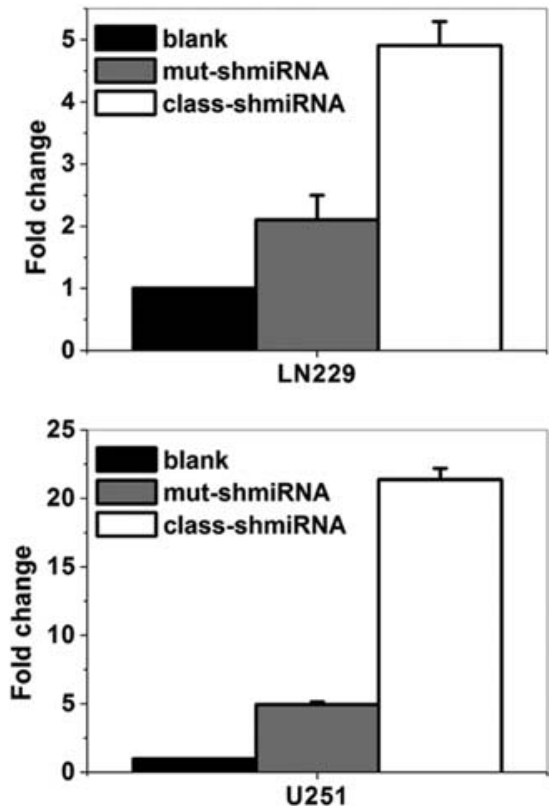

Figure 4. Increase of $\mathrm{p} 27^{\mathrm{kip} 1}$ protein expression induced by adenovirally-encoded shRNAs. (A) Bioinformatics analysis showing the miR-221/222-specific binding sequence of p27 $7^{\mathrm{kip} 1} 3^{\prime} \mathrm{UTR}$. (B) Western blot showing varying levels of p27 $7^{\mathrm{kip} 1}$ expression in LN229 and U251 cells treated with mut-shmiRNA and class-shmiRNA. The bottom row shows the GAPDH loading control. (C) Histograms indicating fold-changes of p27kip 1 protein expression in LN229 and U251 cells.
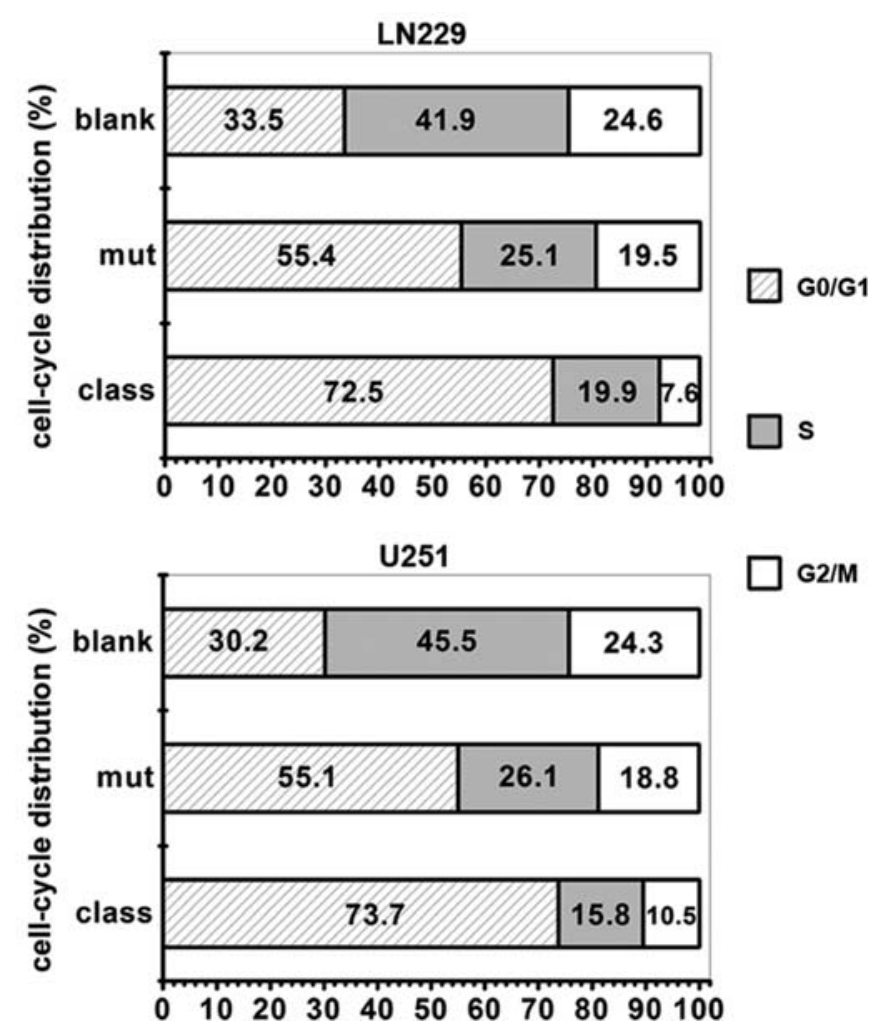

Figure 5. Enhancement of adenovirally encoded shRNAs on cell cycle arrest in $\mathrm{G} 1$ phrase. Cell cycle distribution was analyzed after transduction of shmiRNAs, LN229 and U251 cells were harvested, fixed and stained with propidium iodide.

Increase of p27kipl protein expression by adenovirally encoded shRNAs. The gene encoding the cell cycle inhibitor $\mathrm{p} 27^{\mathrm{kip} 1}$ is a validated target of miR-221/222 in glioblastoma cells
(Fig. 4A). Recent studies have shown that co-repression of miR-221/222 expression might inhibit glioblastoma cell proliferation by a mechanism involving the up-regulation of p2 $7^{\mathrm{kip} 1}$ in vitro and in vivo (9). Based on this information, we hypothesized that mut-shmiR-221/222 and class-shmiR-221/ 222 would affect not only the expression of miR-221/222, but also their function. Indeed, Western blot analysis normalized to GAPDH revealed 2.11 \pm 0.40 -fold and 4.91 \pm 0.38 -fold increases in the level of p2 $7^{\mathrm{kip} 1}$ in LN229 cells treated with Ad-mut-shmiR-221/222 and Ad-class-shmiR-221/222, $4.96 \pm 0.18$-fold and 21.39 \pm 0.83 -fold increases in U251 cells treated with Ad-mut-shmiR-221/222 and Ad-class-shmiR-221/ 222 respectively, compared with blank control cells (Fig. 4B and C).

Enhancement of cell cycle arrest in $G_{1}$ phase by adenovirally encoded shRNAs. To further explore the differential effects of mut-shRNA and class-shRNA on miR-221/222 function via p2 $7^{\mathrm{kip} 1}$ in more detail, we quantified the extent of mut-shRNA or class-shRNA-induced changes to the cell cycle distribution in LN229 and U251 cells by flow cytometry. FCM analysis showed a $21.9 \%$ increase in $\mathrm{G}_{0} / \mathrm{G}_{1}$ phase cells and a $16.8 \%$ decrease in S phase cells in mut-shRNA treated LN229 cells, and a $39.0 \%$ increase in $\mathrm{G}_{0} / \mathrm{G}_{1}$ phase cells and a $22.0 \%$ decrease in S phase in class-shRNA treated LN229 cells. In $\mathrm{U} 251$ cells there was a $24.8 \%$ increase in $\mathrm{G}_{0} / \mathrm{G}_{1}$ phase cells and a $19.4 \%$ decrease in $\mathrm{S}$ phase in the mut-shRNA group, and a $43.5 \%$ increase in $\mathrm{G}_{0} / \mathrm{G}_{1}$ phase cells and a $29.7 \%$ decrease in $\mathrm{S}$ phase in the class-shRNA group. Note that the amount of $\mathrm{G}_{0} / \mathrm{G}_{1}$ phase cells in the class-shmiRNA transduced group was considerably diminished compared with that in the presence of mut-shRNA (LN229: $\chi^{2}=18.377, \mathrm{P}=0.000$; U251: $\left.\chi^{2}=18.377, \mathrm{P}=0.000\right)$. In addition, the level of the $\mathrm{S}$ phase fraction correlated inversely to the $G_{0} / G_{1}$ phase fraction 

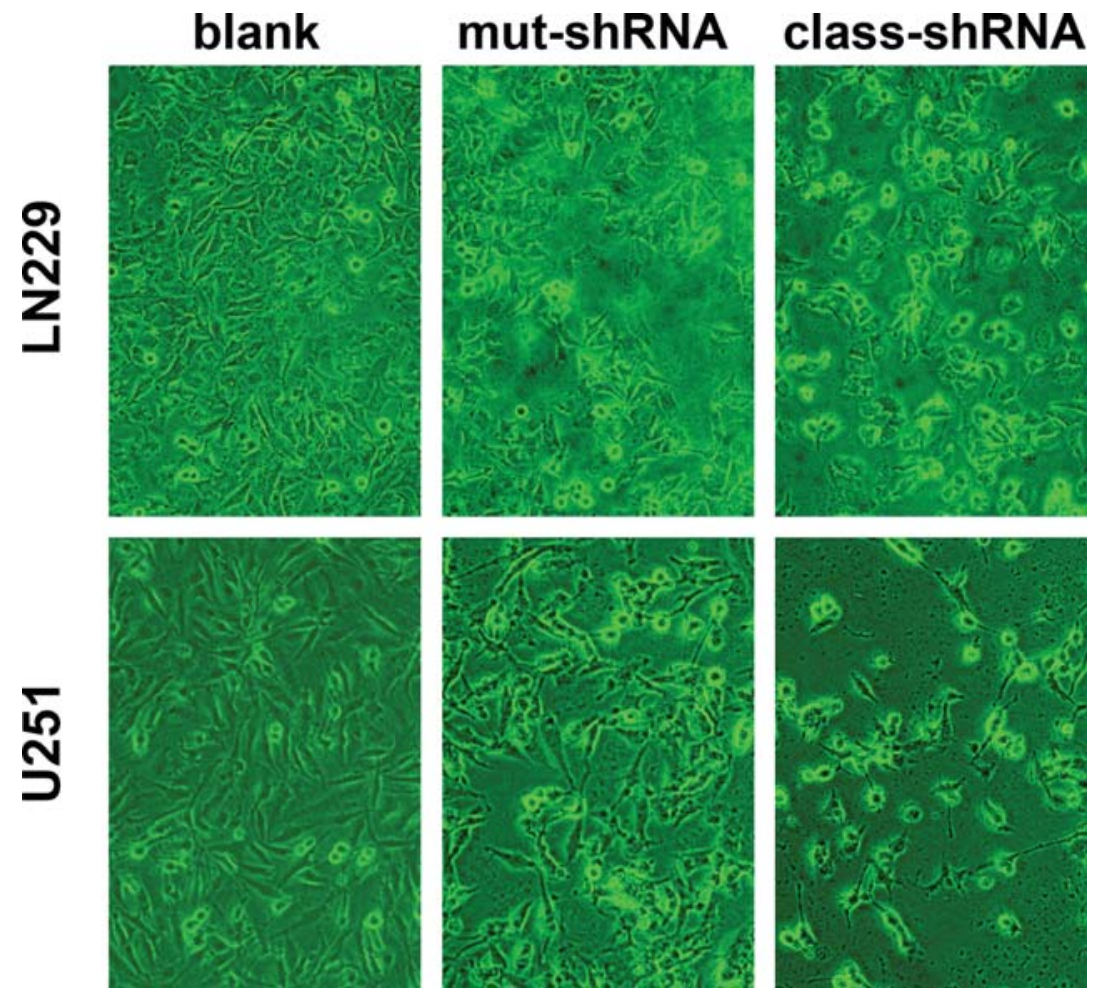

Figure 6. Morphological changes induced by adenovirally encoded shRNAs. The cellular damage was photographed using a phase-contrast microscope in LN229 and U251 cells treated with Ad-mut-shmiR-221/222 or Ad-class-shmiR-221/222 for $48 \mathrm{~h}$.

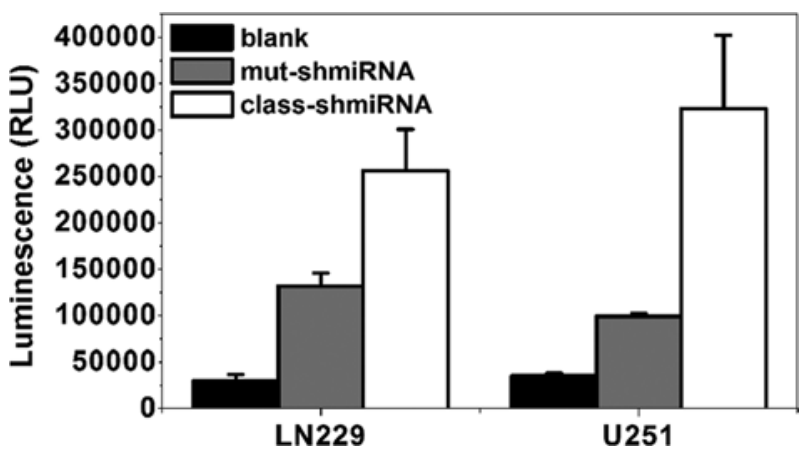

Figure 7. Effects of Ad-mut-shmiR-221/222 and Ad-class-shmiR-221/222induced apoptosis. Cellular apoptosis was determined by measuring activated caspase 3/7 levels using Caspase-Glo 3/7 assay. RLU, relative light units per second.

(LN229: $\left.\chi^{2}=15.909, \mathrm{P}=0.000 ; \mathrm{U} 251: \chi^{2}=15.909, \mathrm{P}=0.000\right)$. These findings are consistent with the proposition that regulation of the $\mathrm{G}_{1}$-to-S phase transition is associated with an increase in $\mathrm{p} 27^{\mathrm{kip} 1}$ protein expression (Fig. 5).

Impact of adenovirally encoded shRNAs on cell apoptosis. To study the shRNAs effects on cell apoptosis, we examined morphological changes in LN229 and U251 cells in the presence of mut-shmiRNA or class-shmiRNA. The cellular damage induced in class-shmiRNA transduced cells was obvious when observed under a phase-contrast microscope, whereas such damage was minimal in mut-shmiRNA transduced cells, compared with blank control cells (Fig. 6). To authenticate this observation, we used the Caspase-Glo 3/7 Assay in these cell lines under the same treatment conditions. Caspase activity, represented by the detected luminescence, was significantly more pronounced in classshmiRNA transduced cells than in mut-shmiRNA transduced cells, compared with blank control cells (LN229: $F=69.262$, $\mathrm{P}=0.000$; $\mathrm{U} 251$ : $\mathrm{F}=43.242, \mathrm{P}=0.000)$. The results indicated that class-shmiRNA caused a distinct increase in apoptosis in gliobastoma cells (Fig. 7).

Prediction of tertiary structures and calculation of free energy of guiding strand:miRNA complexes. To explain the results above, we attempted to detect the free energy state of each complex of guiding strand:miRNA. The free energy state often differs after a change of tertiary structure, and reaching the optimal structure is of primary importance. Using RNAfold, we could produce optimal experimentally determined tertiary structures from the sequences of the complexes and accurately calculate the free energy. RNAfold generated the best solution match for the complexes of target miRNAs and guiding strand, regardless of whether they mutated or classic shRNAs (Fig. 8B, C, F, and G). In addition, the structures were compared with the one formed by a scrambled guiding strand and miR-221 (miR-222) as a negative control (Fig. 8A and E), and the folding of premiR-221 (pre-miR-222) served as a positive control (Fig. 8D and $\mathrm{H})$.

Based on the tertiary structures predicted above, we applied RNAeval to evaluate their corresponding free energy states (Table II). Our results indicated that mutated guiding strand: 
A

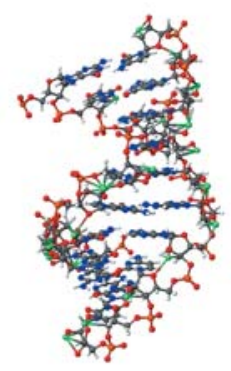

E

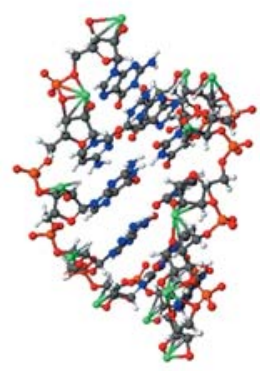

B

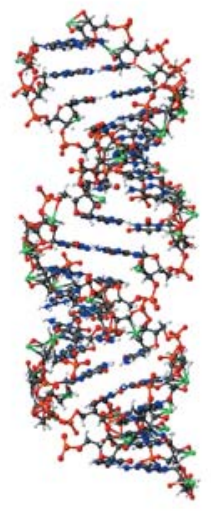

F

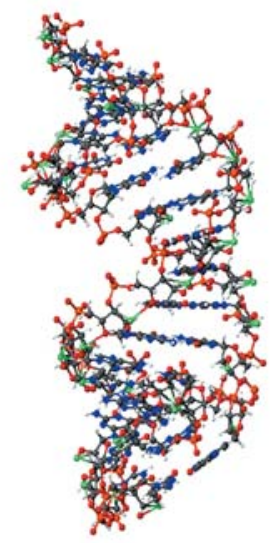

C

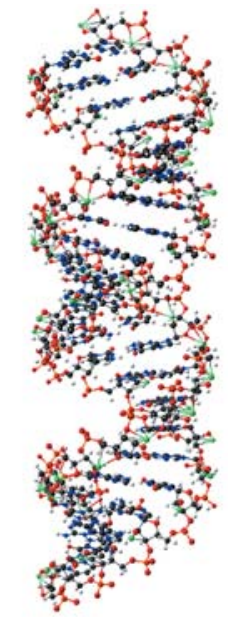

G

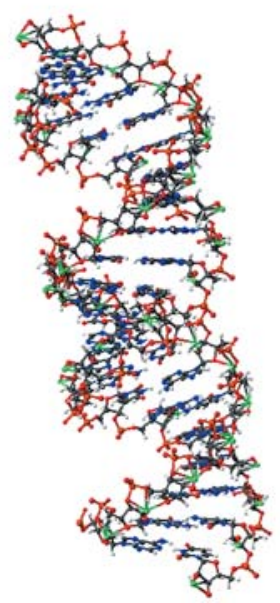

D

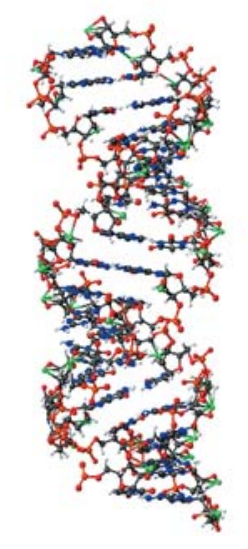

H

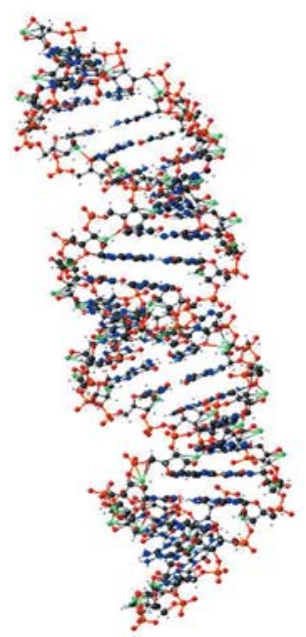

Figure 8. Optimal tertiary structures of complexes formed by the 'guiding strands (GS)' of shRNAs with target miRNAs, predicted by RNAfold. (A) scr-GS221 and miR-221. (B) mut-GS-221 and miR-221. (C) class-GS-221 and miR-221. (D) pre-miR-221. (E) scr-GS-222 and miR-222. (F) mut-GS-222 and miR-222. (G) class-GS-222 and miR-222. (H) pre-miR-222. Scrambled guiding strands were used as negative controls and pre-miRNAs were used as positive controls.

Table II. Free energy states $\left(\mathrm{kcal} \mathrm{mol}^{-1}\right)$ for the structures of complexes formed by the guiding strand of an shRNA with an miRNA.

\begin{tabular}{lcccc}
\hline & scr-shRNA & mut-shRNA & class-shRNA & pre-miRNA \\
\hline miR-221 & -9.6 & -32.7 & -42.1 & -47.2 \\
$\operatorname{miR}-222$ & -4.2 & -32.7 & -41.5 & -54.1 \\
\hline
\end{tabular}

miRNA complexes were less stable than class guiding strand: miRNA complexes as their free energy states were ranked higher.

\section{Discussion}

Our evolving understanding of the molecular pathways that are important for carcinogenesis has created opportunities for cancer therapy using RNAi technology to target the key molecules within these pathways. For example, Tomlinson et al used retrovirus-mediated delivery of shRNAs to knock down
S249C mutant FGFR3 as a potential therapeutic target in bladder cancer (11), while Scherr et al applied lentiviralmeditated delivery of shRNAs to modulate the aberrant expression of the polycistronic miR17-92 miRNA cluster in early phase of chronic myeloid leukemia (CML) (6). These preclinical studies confirmed that RNAi techniques could be used to silence cancer-related targets, including oncogenes and miRNAs. In our study, to suppress the specific miRNAs that are aberrantly expressed in glioblastama cells, we used an adenoviral vector to deliver the shRNA expression cassette. Compared with lenti- and retrovirus, adenoviral vectors have 
a high transduction efficiency, are capable of containing DNA inserts up to 8 kilobases, have extremely high viral titers (on the order of $10^{10}-10^{13}$ ), and infect both replicating and differentiated cells. Also, as they are deficient in integration, they can avoid the mutagenic effects caused by random integration into the host genome, and thus constitute a powerful and valuable vector system.

Cancer is a kind of polygenic disease; therefore, it would be convenient if some of the implicated genes could be modulated simultaneously. Accordingly, we recently generated an adenoviral vector embedded with three shRNAs to suppress related oncogenes of gastric adenocarcinoma and glioblastoma cells and obtained significant proliferation and invasion inhibition effects (12). In the present study, we produced an adenoviral vector embedded with two shRNAs targeting aberrantly expressed miRNAs in glioblastama cells simultaneously.

With this vehicle, we introduced a series of miR-221/222 shRNAs into glioblastoma cells. Different shRNAs produced distinct semi-quantitative results in the reduction of miR221/222 expression. Semi-quantitative modulation of gene expression has been achieved by Scherr et al in miRNA (6) and Hemann et al in Tp53 (13). Lentiviral vectors of Scherr et al encoded EGFP (green fluorescence protein) as a reporter gene driven by a viral SFFV (spleen focus forming virus)-LTR promoter, and the transduced cell lines could be sorted according to their EGFP fluorescence intensity, which correlated directly and semi-quantitatively with reduction of miRNA function and concomitant increase of target protein expression (6). On the other hand, Hemann et al designed shRNAs by altering their position in the backbone of vectors and targeting a distinct sequence in Tp53 to acquire similar results. In our study, besides the classic shRNA, we produced another miR-221/miR-222 shRNA by mutating the 3'-end bases of the shRNAs in the antisense sequence (guiding strand) that were complementary to the target miRNA 5'-seed region, and assessed their ability to influence miR-221/222 levels and activity.

Transient transduction of the mutated and classic shRNA vectors into cultured cells resulted in varying levels of miR221/222 suppression: the classic shRNA achieved a greater reduction in miR221/222 levels than the mutated shRNA, as determined by qRT-PCR. In addition, target protein expression can be regulated by miRNAs, as demonstrated for miR-221/ 222 and its experimentally validated target p27kip1 (9), and the change in $\mathrm{p} 27^{\mathrm{kip} 1}$ protein levels correlated inversely with miR-221/222 levels in our study. The p27kip1 (CDKN1B) protein belongs to the Cip/Kip family of cyclin dependent kinase $(\mathrm{CDK})$ inhibitor proteins, and thus controls cell cycle progression at the $\mathrm{G}_{1}$ phase (14). Consequently, we performed cell cycle analysis by flow cytometry, and revealed differences among the miR-221/222 vectors. Consistent with the varied ability of shRNAs to reduce miR-221/222 levels, the classic shRNA produced a greater $\mathrm{G}_{1}$ phase enhancement compared to the mutated shRNA. To further prove the differential response to the mutated and classic shRNA vectors, we explored the potential role of shRNA vectors in inducing apoptosis. We monitored their effects on cell morphology under a phase-contrast microscope and used a Caspase-Glo $3 / 7$ assay, and similar results were obtained. Thus, we demonstrated that this novel strategy allows modulation of miRNAs in a semi-quantitative manner.

The purpose of the nucleotide cyclic motifs (NCM)-based study was to expand our knowledge on the intricate mechanisms of our shRNA semi-quantitative control of miRNA expression and function by focusing on the structural determinants that influenced the stability of guiding strand: target miRNA complex in terms of their free-energy states. More importantly, the prediction of the optimal structures of complexes is the fundamental for the correct free energy calculation.

NCM is a first-order object to represent nucleotide relationships in structured RNAs. It provides more context-dependent information, compared to the classical approach to RNA modeling such as the thermodynamic approach, which considers A:U and G:C Watson-Crick as well as G:U wobble base pairs, i.e. only those interactions where the nucleotides are facing each other (10). Accordingly, Parisien et al (10) implemented two algorithms that systematically assign the different NCMs to each segment of the sequence and select the most probable pair based on its frequency in known structures, and then assemble the set of selected NCMs, taking into account the constraints that are found in known structures.

Similarly, we introduced the modeling algorithm RNAfold to unravel the structure of guiding strand:target miRNA complexes. The free-energy was then calculated based on the predicted complexes by another algorithm, RNAeval. In summary, mutation of shRNA weakens the stability of the guiding strand:target miRNA complex, thus reduces the suppressing ability of shRNA, leading to the modulation of miRNA in a semi-quantitative manner compared with classic shRNAs.

In conclusion, using adenovirus-mediated shRNAs, tumorrelated multiple miRNAs could be repressed simultaneously. By applying mutation strategy, we yielded various levels of modulation of miRNA expression and function. As the study of the structural aspects shows, the mutation enhances the free-energy state to influence the stability of the guiding strand:target miRNA complex, which may be of critical importance in understanding these intricate mechanisms.

\section{Acknowledgments}

This study was supported by the China National Natural Scientific Found (30971136 and 30772231), Tianjin Science and Technology Committee (07ZCGHHZ01000), and the Program for New Century Excellent Talents in University (NCET-07-0615). The authors are grateful to Xin-yan Wang (School of Architecture and Fine, Dalian University of Technology, Liaoning, China) for his outstanding technical illustration support in Figs. 1 and 2.

\section{References}

1. Lee RC, Feinbaum RL and Ambros V: The C. elegans heterochronic gene lin-4 encodes small RNAs with antisense complementarity to lin-14. Cell 75: 843-854, 1993.

2. Karginov FV, Conaco C, Xuan Z, et al: A biochemical approach to identifying microRNA targets. Proc Natl Acad Sci USA 104: 19291-19296, 2007. 
3. Cui Q, Yu Z, Purisima EO, et al: Principles of microRNA regulation of a human cellular signaling network. Mol Syst Biol 2: 46, 2006.

4. Bhatti I, Lee A, Lund J, et al: Small RNA: a large contributor to carcinogenesis? J Gastrointest Surg 13: 1379-1388, 2009.

5. Paddison PJ, Caudy AA, Bernstein E, et al: Short hairpin RNAs (shRNAs) induce sequence-specific silencing in mammalian cells. Genes Dev 16: 948-958, 2002.

6. Scherr M, Venturini L, Battmer K, et al: Lentivirus-mediated antagomir expression for specific inhibition of miRNA function. Nucleic Acids Res 35: e149, 2007.

7. Ciafrè SA, Galardi $\mathrm{S}$, Mangiola $\mathrm{A}$, et al: Extensive modulation of a set of microRNAs in primary glioblastoma. Biochem Biophys Res Commun 334: 1351-1358, 2005.

8. Zhou X, Ren Y, Moore L, et al: Downregulation of miR-21 inhibits EGFR pathway and suppresses the growth of human glioblastoma cells independent of PTEN status. Lab Invest 90: $144-155,2010$

9. Zhang C, Kang C, You Y, et al: Co-suppression of miR-221/ 222 cluster suppresses human glioma cell growth by targeting p27 ${ }^{\mathrm{kip} 1}$ in vitro and in vivo. Int J Oncol 34: 1653-1660, 2009.
10. Parisien M and Major F: The MC-Fold and MC-Sym pipeline infers RNA structure from sequence data. Nature 452: 51-55, 2008.

11. Tomlinson DC, Hurst CD and Knowles MA: Knockdown by shRNA identifies S249C mutant FGFR3 as a potential therapeutic target in bladder cancer. Oncogene 26: 5889-5899, 2007.

12. Fu Y, Zhang Q, Kang C, et al: Inhibitory effects of adenovirus mediated COX-2, Akt1 and PIK3R1 shRNA on the growth of malignant tumor cells in vitro and in vivo. Int J Oncol 35: 583-591, 2009

13. Hemann MT, Fridman JS, Zilfou JT, et al: An epi-allelic series of p53 hypomorphs created by stable RNAi produces distinct tumor phenotypes in vivo. Nat Genet 33: 396-400, 2003.

14. Toyoshima $\mathrm{H}$ and Hunter $\mathrm{T}$ : p27, a novel inhibitor of $\mathrm{G} 1$ cyclin-Cdk protein kinase activity, is related to p21. Cell 78: 67-74, 1994. 